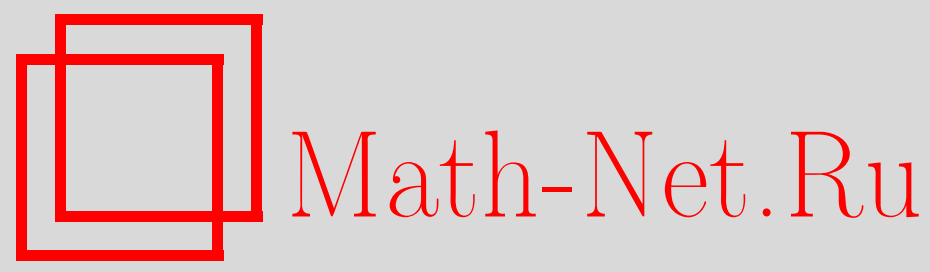

В. П. Маслов, А. Э. Рууге, Об одном симметрическом представлении уравнения для квантового хаоса, Матем. заметки, 1997, том 62, выпуск 6, 940-941

DOI: https://doi.org/10.4213/mzm1685

Использование Общероссийского математического портала Math-Net.Ru подразумевает, что вы прочитали и согласны с пользовательским соглашением http://www.mathnet.ru/rus/agreement

Параметры загрузки:

IP : 3.93.64.190

26 апреля 2023 г., $17: 34: 15$

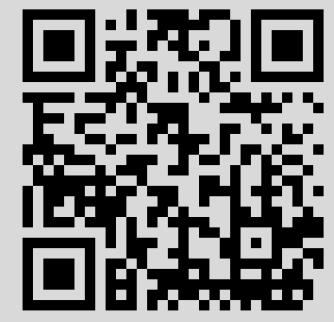




\section{ОБ ОДНОМ СИММЕТРИЧЕСКОМ ПРЕДСТАВЛЕНИИ УРАВНЕНИЯ ДЛЯ КВАНТОВОГО ХАОСА}

\section{В.П. Маслов, А. Э. Рууге}

В работе [1] предъявляется уравнение для квантового хаоса вида

$$
i \frac{\partial}{\partial \tau}\left(1+\frac{i h}{2} \frac{\partial}{\partial z}\right) \varphi=\lambda \varphi\left(\bmod h^{2}\right), \quad \varphi \in S_{h}
$$

на гладком многообразии

$$
M=\left\{(p, x) \in \mathbb{R}^{2 k} \mid H(p, x)=E^{0}\right\}, \quad E^{0}=\mathrm{const},
$$

где $H: \mathbb{R}^{2 k} \rightarrow \mathbb{R},\left.|\nabla H|\right|_{M} \neq 0 ; h>0, h \rightarrow 0 ; S_{h}-$ класс функций

$$
S_{h}=\left\{\varphi(\alpha, h) \mid \varphi(\alpha, h)=\sum_{r=1}^{N} \varphi_{r}(\alpha) h^{r}, \varphi \in C^{\infty}(M), N<\infty\right\}
$$

$\partial / \partial \tau-$ ограничение гамильтонова векторного поля

$$
\frac{d}{d \tau}=\sum_{j=1}^{k}\left(\frac{\partial H}{\partial p_{j}} \frac{\partial}{\partial x_{j}}-\frac{\partial H}{\partial x_{j}} \frac{\partial}{\partial p_{j}}\right)
$$

на $M ; \partial / \partial z$ - ограничение на $M$ коммутатора $d / d \tau$ с произвольньм векторным полем $d / d E$ в окрестности $M$, удовлетворяюшим условию $d H / d E=1$ (например, $d / d E=$ $\left.\left(1 /|\nabla H|^{2}\right) \cdot(\nabla H, \nabla)\right)$ :

$$
\left.\frac{\partial}{\partial z} \stackrel{\text { def }}{=}\left[\frac{d}{d \tau}, \frac{d}{d E}\right]\right|_{M} .
$$

Уравнение (1) имеет следуюший смысл. Рассмотрим квантовомеханическую систему c $k$ степенями свободы, гамильтониан $\widehat{H}$ которой имеет вид

$$
\widehat{H}=H\left(-i h \frac{\stackrel{\omega}{\partial}}{\partial x}, \stackrel{\omega}{x}\right)
$$

Оператор $\widehat{H}$ является инфинитезимальным генератором унитарной группы, определяющей динамику данной системы. Если гамильтонов фазовьй поток, порожденный $H(p, x)$, является эргодическим на $M$, то указанная динамика назьвается квантовым хаосом. Предположим, что спектр $\widehat{H}$ дискретен в некоторой окрестности $\left(E^{0}-\delta, E^{0}+\delta\right)$, $\delta=$ const. В этом случае значения $\lambda$, определяемые из $(1)$, равны с точностью $O\left(h^{3}\right)$ разностям собственных значений $E_{n}$ оператора $\widehat{H}$, принадлежащих одной серии [2] и лежащих вблизи уровня $E^{0}$ :

$$
E_{n}-E_{m}=h \lambda+O\left(h^{3}\right), \quad E_{n}, E_{m} \rightarrow E^{0}, \quad h \rightarrow 0 .
$$

Работа выполнена при финансовой поддержке фонда INTAS, грант № 94-2763. 
Строгая формулировка соответствующей теоремы приведена в [1].

Определим на многообразии $M$ инвариантную относительно векторного поля $\partial / \partial \tau$ меру $d \nu$ при помощи соотношения

$$
\left.\int \varphi\right|_{M} d \nu=\int \delta\left(H(p, x)-E^{0}\right) \varphi(p, x) d p d x \quad \forall \varphi \in C_{0}^{\infty}\left(\mathbb{R}^{2 k}\right) .
$$

Заметим, что в случае, когда фазовый поток $H(p, x)$ эргодичен на $M$, такая мера определена однозначно с точностью до произвольного постоянного множителя.

Определим на пространстве гладких функций на $M$ скалярное произведение

$$
(\varphi, \Phi)=\int \varphi^{*} \Phi d \nu, \quad \varphi, \Phi \in C^{\infty}(M) .
$$

ПРЕДЛОЖЕНИЕ 1. Векторное поле Ә/Әz представимо в виде

$$
\frac{\partial}{\partial z}=-\left.\left(\frac{d}{d E}^{*} \cdot 1\right)\right|_{M} \frac{\partial}{\partial \tau}+\frac{\partial}{\partial w}
$$

где $\partial / \partial ш$ удовлетворяет условию

$$
\left(\varphi, \frac{\partial \Phi}{\partial w}\right)=-\left(\frac{\partial \varphi}{\partial w}, \Phi\right)
$$

$(d / d E)^{*}$ - оператор, сопряжсеный $\kappa d / d$ Е относительно канонической меры $d p d x$.

Пусть $\varphi$ и $\lambda$ удовлетворяют (1). Рассмотрим преобразование

$$
\psi=\left(1+\frac{i h}{4} \frac{\partial}{\partial w}\right) \varphi .
$$

Заметим, что $\psi \in S_{h}$, при этом

$$
\varphi=\left(1-\frac{i h}{4} \frac{\partial}{\partial w}\right) \psi\left(\bmod h^{2}\right) .
$$

Из (1) вытекает следующее соотношение на $\psi$ :

$$
\begin{aligned}
\widehat{\mathscr{L}} \psi & \stackrel{\text { def }}{=}\left\{i \frac{\partial}{\partial \tau}+\frac{h}{2}\left(\left.\frac{\partial}{\partial \tau}\left(\frac{d}{d E}^{*} \cdot 1\right)\right|_{M} \frac{\partial}{\partial \tau}-\frac{1}{2}\left[\frac{\partial}{\partial \tau} \frac{\partial}{\partial w}+\frac{\partial}{\partial w} \frac{\partial}{\partial \tau}\right]\right)\right\} \psi \\
& =\lambda \psi\left(\bmod h^{2}\right), \quad \psi \in S_{h} .
\end{aligned}
$$

Соотношения (1) и (3) эквивалентны.

ПРЕДЛОЖЕнИЕ 2. Оператор $\widehat{\mathscr{L}}$ симметричен относительно скалярного произведения (2).

\section{СПИСОК ЦИТИРОВАННОЙ ЛИТЕРАТУРЫ}

1. Maslov V. P. // Russian J. Math. Phys. 1996. V. 4. № 3. P. 377-392. 2. Maslov V. P. // Russian J. Math. Phys. 1996. V. 4. № 4. P. 529-534.

Московский государственный университет им. М. В. Ломоносова 